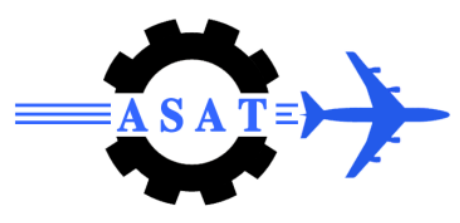

\title{
Buried Object Detection Based on Multivariate Latent Methods
}

\author{
$\left\{\text { K. A. Ghamry }{ }^{*} \text { W. M. Hussein, M. Hegazy, H. M. Mahgoub }\right\}^{\dagger}$
}

\begin{abstract}
In this paper, wideband ground penetrating radar (GPR) system and a proposed frequency domain data analysis technique are presented for the detection of shallow buried objects such as anti-personal (AP) land mines. The GPR system uses one antenna for transmitting and for receiving and. it operates from $9 \mathrm{GHz}$ to11 GHz. This system is able to acquire, save and analyze the data in the frequency domain. Experiments were conducted on metallic and plastic AP mines under different depths. Principal Component Analysis (PCA) has been used for processing the data. This technique is effective for the clustering of different groups. Results show that the proposed technique can successfully differentiate between metallic and plastic mines-like targets buried in dry soil.
\end{abstract}

Keywords: Buried object, monitoring, multivariate analysis, PCA.

\section{Introduction}

In the last century, specifically in its 4th decade in the World War II, more than 22 millions of mines have been planted in Egypt [1] it is important to locate these mines that can potentially cause massive number of deaths and casualties. Because of this the process of detecting and removing mines, called demining, is particularly important. But it's extremely dangerous; one deminer has been killed for every 2,000 mines removed, with even more civilian victims. The cost to purchase and lay a typical antipersonnel mine ranges from $\$ 3$ to $\$ 30$, while the cost to remove a single mine ranges from $\$ 300$ to $\$ 1000$ [1]. In 1994, approximately 200,000 mines were removed, while two million new mines were planted. Many experts believe that it would take more than ten centuries to remove every mine in the world with the current clearance rate, even if no additional mines were planted [2].

Because mines can be made of both metallic and nonmetallic materials, detection using only conventional metal detectors is subjected to many false alarms due to the presence of small fragments of munitions resulted from previous wars.

To meet the strict requirements for humanitarian demining, various techniques in the area of sensor physics, signal processing, and robotics have been studied during the last decade.

Most mine detection techniques consist of sensor, signal processing, and decision processes. For the sensor part, metal detectors, ground penetration radar (GPR), infrared (IR), and ultrasound (US) sensors are used.

Because of the capabilities of good penetration and depth resolution as well as detecting both metallic and nonmetallic objects, ground penetrating radar (GPR) [3] has been considered as

\footnotetext{
*kha_shaaban@yahoo.com

${ }^{\dagger}$ Egyptian Armed Forces, Cairo, Egypt.
} 
an effective technology for land mine detection. A GPR system consists of a transmitter for emitting electromagnetic wave to the inspection land and a receiver for collecting the returned signal from which the decision of whether there is a mine is made. But, detecting land mineswith GPR is still a difficult task because strong reflections from the ground surface and other gradients in the land dominate the mine response specifically when the mines are deeply buried. On the other hand, it could be difficult to differentiate a land mine and a clutter object even if the ground bounce is successfully removed. As a result, signal processing is an important step for rendering the GPR sensor outputs to increase probability of detection and reducing false alarm rate.

For the signal processing part, many signal processing algorithms have been proposed for GPR data. Some of them are the hidden Markov model (HMM) [4] that is designed to detect the hyperbolic signature produced from a land mine, the least mean square (LMS) algorithm [5] that detects anomaly in the soil background, and the principal component analysis (PCA) [6] that is used to model the soil background, and that we used in this paper.

In this work, a statistical multivariate technique called Principal Component Analysis (PCA) is applied to detect buried object as a health monitoring technique in order to save effort and money spent through traditional techniques used for mine detection.

Most monitoring techniques presently available describe the variation of one or a limited number of sensory data using a heuristic approach which gives limited information about machine condition. Among these techniques, artificial neural network ANNs and its combination with other methods have been the most predominant to date $[11,12]$. ANNs techniques are characterized as black box approaches which model the relation between different variables to a desired response without giving any information about what happens inside the process.

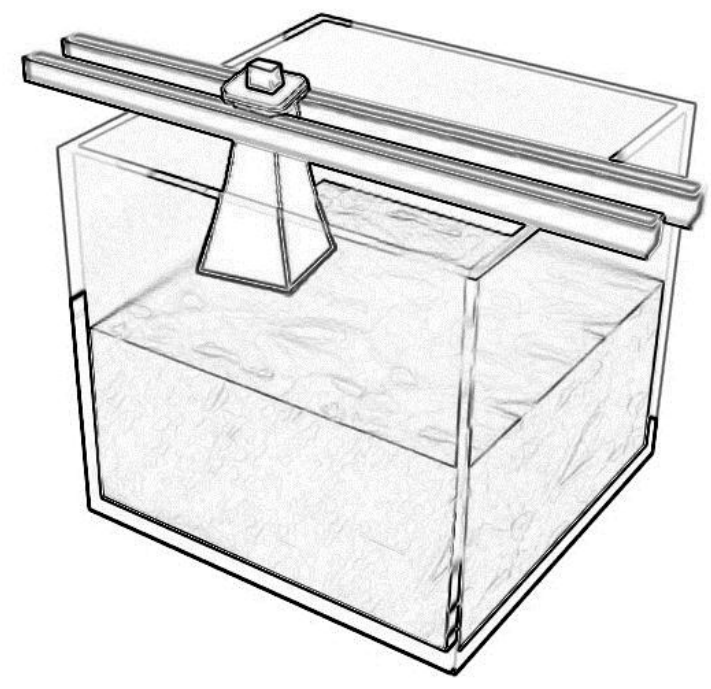

Fig. 1 Scheme drawing for detecting buried object experimentally

Also some of these approaches are susceptible to missing data due to sensor reliability issues, and some applications use many charts to monitor individual process. In addition, issues associated with co linearity and dimensionality needs to still be specifically addressed in these techniques. Looking for a generic approach to solve many of these limitations can be addressed through the use of multivariate statistical analysis, a data driven modeling approach 
[13]. Multivariate statistical process control (MSPC) [14], takes a different approach as compared to the other methods mentioned previously. The most fundamental difference with this approach is that the model is based on non-causal empirical correlations extracted from normal plant operating data when only common cause variation exists. The simplicity of this approach is that there is no need for a fundamental model of the system and only data from normal production needs to be used, which is generally available in some form at most factories. Among the approaches used in multivariate analysis are: a projection method which called principal component analysis (PCA) $[15,16]$ and projection to latent structure (PLS) [17]. Many applications of these two techniques have been successfully applied in other fields of process monitoring [11, 18-23] ranging from batch to continuous processes. Principal component analysis (PCA) is the most widely used data-driven technique for process monitoring which has been heavily studied and applied to industrial systems over the past decade. PCA is an optimal dimensionality reduction technique in terms of capturing the variance of the data, and it accounts for correlations among variables. The lower-dimensional representations of the data produced by PCA can improve the proficiency of detecting and diagnosing faults using multivariate statistics. The principal components span a low dimensional subspace used for analysis. The details of linear PCA can be found elsewhere [24].

\section{Multivariate PCA Basic Theory}

Principal component analysis decomposes the variance and covariance structure of a data matrix by defining linear combinations of the columns in the original matrix. Moreover, PCA extracts information from data sets by computing a smaller data set and other summary information that adequately captures most of the underlying features from the larger data set. The point that needs to be stressed is that the data can be reduced to a size which is more manageable but contains the features that are often of interest. A mathematical description of PCA follows - only a basic knowledge of linear algebra is required to understand this description. Let the original data set be represented by the matrix, $\mathrm{X}$, with $\mathrm{N}$ rows and $\mathrm{K}$ columns. PCA extracts a score matrix, $\mathrm{T}$, and a loading matrix, $\mathrm{P}$, from $\mathrm{X}$. These matrices have the following dimensions:

\section{$\mathrm{X}: \mathrm{N} \times \mathrm{K} \quad \mathrm{T}: \mathrm{N} \times \mathrm{A} \quad \mathrm{P}: \mathrm{K} \times \mathrm{A}$}

The matrices $\mathrm{T}$ and $\mathrm{P}$ cannot have more columns than the original data set, as $\mathrm{A} \leq \mathrm{K}$; the reduction in the data size is achieved by choosing A to be smaller than $\mathrm{K}$.

We call the first column of $\mathrm{T}$ and $\mathrm{P}$ by their shorter forms, $\mathrm{t}_{1}$ and $\mathrm{p}_{1}$ respectively. The lower case letters indicate that these are vectors, upper case letters indicate matrices.

Extracting only one principal component (that is, a single score vector and loading vector) gives: $\mathrm{X}=\mathrm{t}_{1} p_{1}^{T}+\mathrm{E} 1$ and extracting a second principal component:

$\mathrm{X}=\mathrm{t}_{1} p_{1}^{T}+\mathrm{t} 2 p_{2}^{T}+\mathrm{E} 2$

We continue in this manner until we extract A principal components and then group these score and loading vectors to form matrices $\mathrm{T}$ and $\mathrm{P}$ :

$\mathrm{T}=\left[\mathrm{t}_{1} \mathrm{t}_{2} \ldots \mathrm{t}_{\mathrm{A}}\right]$ and $\mathrm{P}=\left[\mathrm{p}_{1} \mathrm{p}_{2} \ldots \mathrm{p}_{\mathrm{A}}\right]$ 
More compactly then: $\mathrm{X}=\mathrm{TP}^{\mathrm{T}}+\mathrm{E}_{\mathrm{A}}$, If we compute as many principal components as there are columns in the data set $\mathrm{X}$, then we can directly write that $\mathrm{X}=\mathrm{TP}^{\mathrm{T}}$, but in this case the amount of data that we have to deal with in $\mathrm{T}$ and $\mathrm{P}$ is the same as what we started with. The only difference is that it is in a more useful form. Specifically, the principal components are orthogonal to each other so that information captured in principal component 1 is not repeated in any subsequent components. We can also monitor statistics on the residual matrices, $\mathrm{E}_{\mathrm{i}}$, to ensure that all significant information of interest has been extracted by the principal components. What remains now is for us to actually compute the columns of $\mathrm{T}$ and $\mathrm{P}$. There exists a very fast algorithm for computing these principal components when given a data set that has many more rows than columns, $\mathrm{N} \gg \mathrm{K}$. Consequently we will only mention and deal with this method. The eigenvectors of the real symmetric matrix $\mathrm{X}^{\mathrm{T}} \mathrm{X}$ give us exactly the loading matrix $\mathrm{P}$. This is the loading matrix obtained by extracting all principal components.

Once we have $\mathrm{P}$ we use the last line of the following relationship to compute $\mathrm{T}$ :

$\mathrm{TP}^{\mathrm{T}}=\mathrm{X} \quad \mathrm{TP}^{\mathrm{T}} \mathrm{P}=\mathrm{XP} \quad \mathrm{T}=\mathrm{XP}$

This works because $X^{T} X$ is a real symmetric matrix and the eigenvectors from such a matrix have the property that $\mathrm{P}^{\mathrm{T}} \mathrm{P}=\mathrm{I}$, with $\mathrm{I}$ being an identity matrix. The speed of this algorithm stems from the fact that the eigenvectors are computed on a very small matrix of size $\mathrm{K} \times \mathrm{K}$ and that we do not have to use the original $\mathrm{N} \times \mathrm{K}$ matrix. Most eigenvector calculation routines also provide the eigenvalues. The largest eigenvalue is associated with the first principal component, the second largest eigenvalue with the second component, and so on. A more in-depth discussion, which also highlights some geometric concepts of PCA, can be found in [9]. The algorithm used to calculate the PCA is the NIPALS (Nonlinear Iterative Partial Least Squares) algorithm [10].

\section{Experimental Setup}

Figures show the schematic and a photograph, respectively, of the experimental setup used to validate the proposed method. The main feature of the test rig is to perform integrated components of electronics, and computer interface structure, with high computational capacity and good software programmability. The sand bin $(35 \times 32 \times 30 \mathrm{~cm})(\mathrm{LxWxH})$ filled with sand represents the tested land which is planted with metal and/or plastic mine-like target. A microwave antenna working in the frequency range of $(8-12 \mathrm{GHz})$ is installed on a wooden carrier on the top of the sand bin to transmit and receiving the microwave to the HP (8510C) vector network analyzer which declare the data curve between the microwave frequency which adjusted between $(9-11 \mathrm{GHz})$ and the power reflected in $(\mathrm{db})$ which is used to determine whether there is a buried object or not trying to differentiate between the metal or plastic mine-like target. This is done by interpreting these data by the "SIMCA-P+" software.

The sand bin is divided by 16 equal portions $(4 \times 4)$ the antenna is laid on each portion, with 6 $\mathrm{cm}$ height from the sand surface, emitting and receiving microwave to detect buried mine-like target in each portion, recording these data on a floppy disk by the network analyzer in order to send it to the computer for the implementation. 


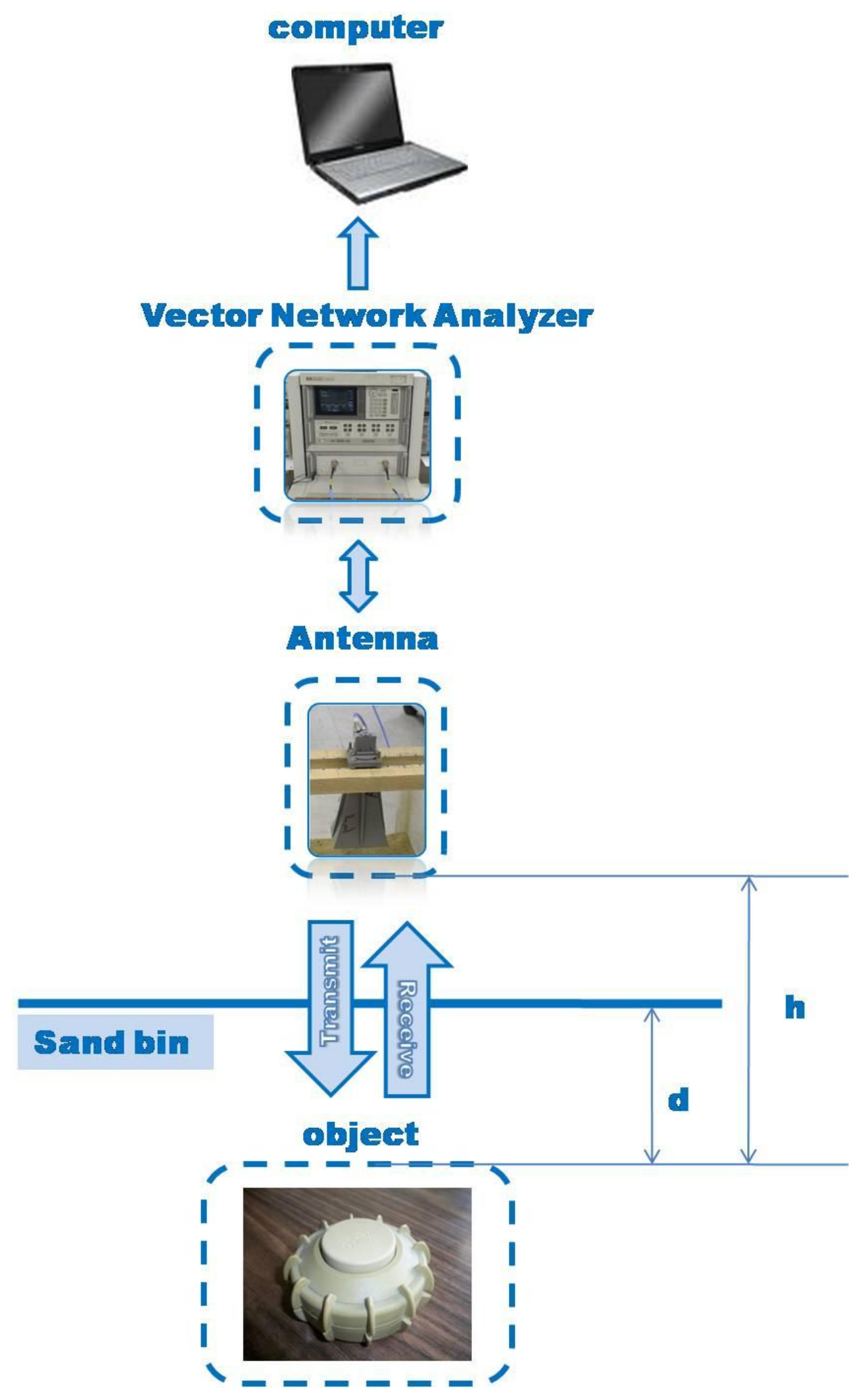

Fig. 2 Schematic of buried object location experimentally 


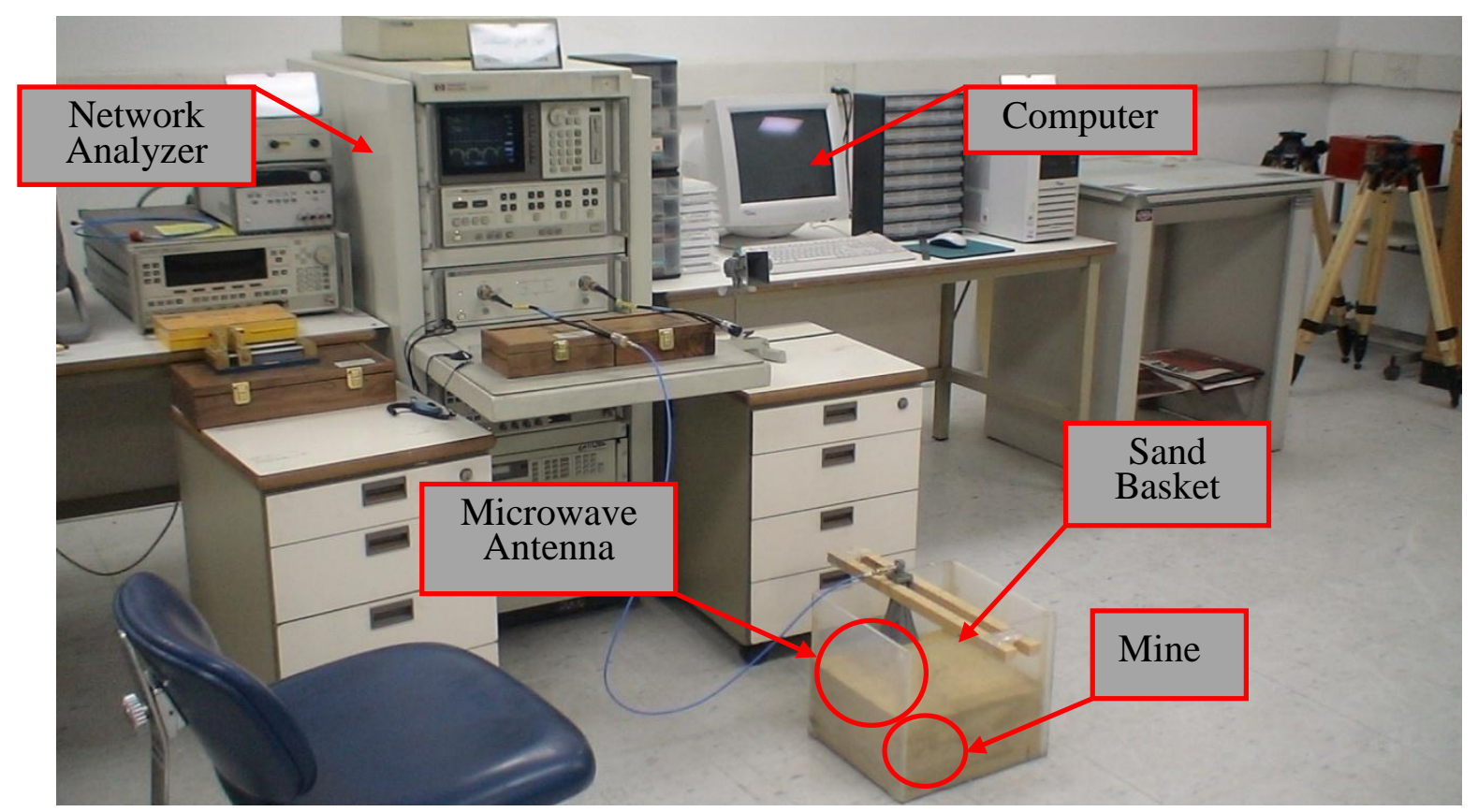

Fig. 3 Experimental setup

\section{Model Building, Configuration and Results}

The multivariate PCA model is a linear model given in matrix form by: $X=\mathrm{TP}^{\mathrm{T}}+\mathrm{E}_{\mathrm{A}}$. However, there exist non-linear PCA versions even by augmenting the original matrix with the non-linear factors or by building a non-linear relation between score factors [10]. To build the model using microwave frequency data, let $\mathrm{X}$ include variables containing the powerfrequency reflections at different depths.

There are different approaches to deal with the available data depending on their type. The simplest way is to use only normal data (empty sand) based on common cause variation and build the model, then use the model to detect any shift (buried object) that will violate the correlation structure established between the variables.

In this section, experimental data are analyzed using PCA. This approach is intended to analyze power-frequency reflection data from the 16 and 208 positions to construct detection system then tested with buried metallic and plastic mines-like targets.

First, all data were mean centered and scaled to unit variance. Second, control limits in the latent space were established using F-distribution based on reference distribution provided by the dataset [18]. Also, $\mathrm{t} 1$ and $\mathrm{t} 2$ are the first two principal components that capture most of the variance in the $\mathrm{X}$ matrix. Figure 4 illustrates how the score plot is built for a simple case 3variables and 2 -scores. After determining the direction of maximum variation by iterative steps and get the second orthogonal direction by the same way after subtracting the first component, we rotate the new plane determined by the new score variables $t_{1}$ and $t_{2}$ and then monitor the change of the new observations in the reduced dimensional space. 


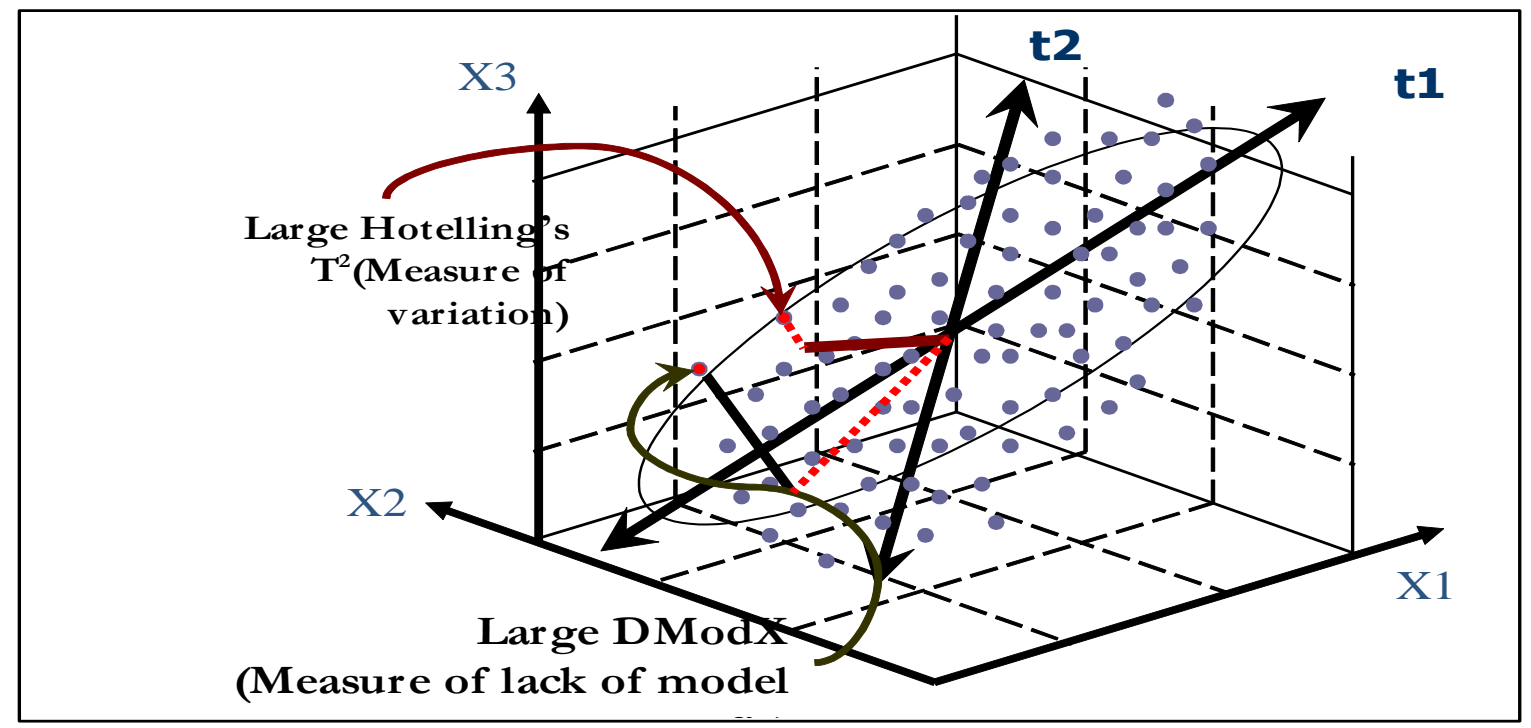

Fig. 4 Establishing score plot for three variables.

A general scheme of the simulated detection system is shown in figure 5.
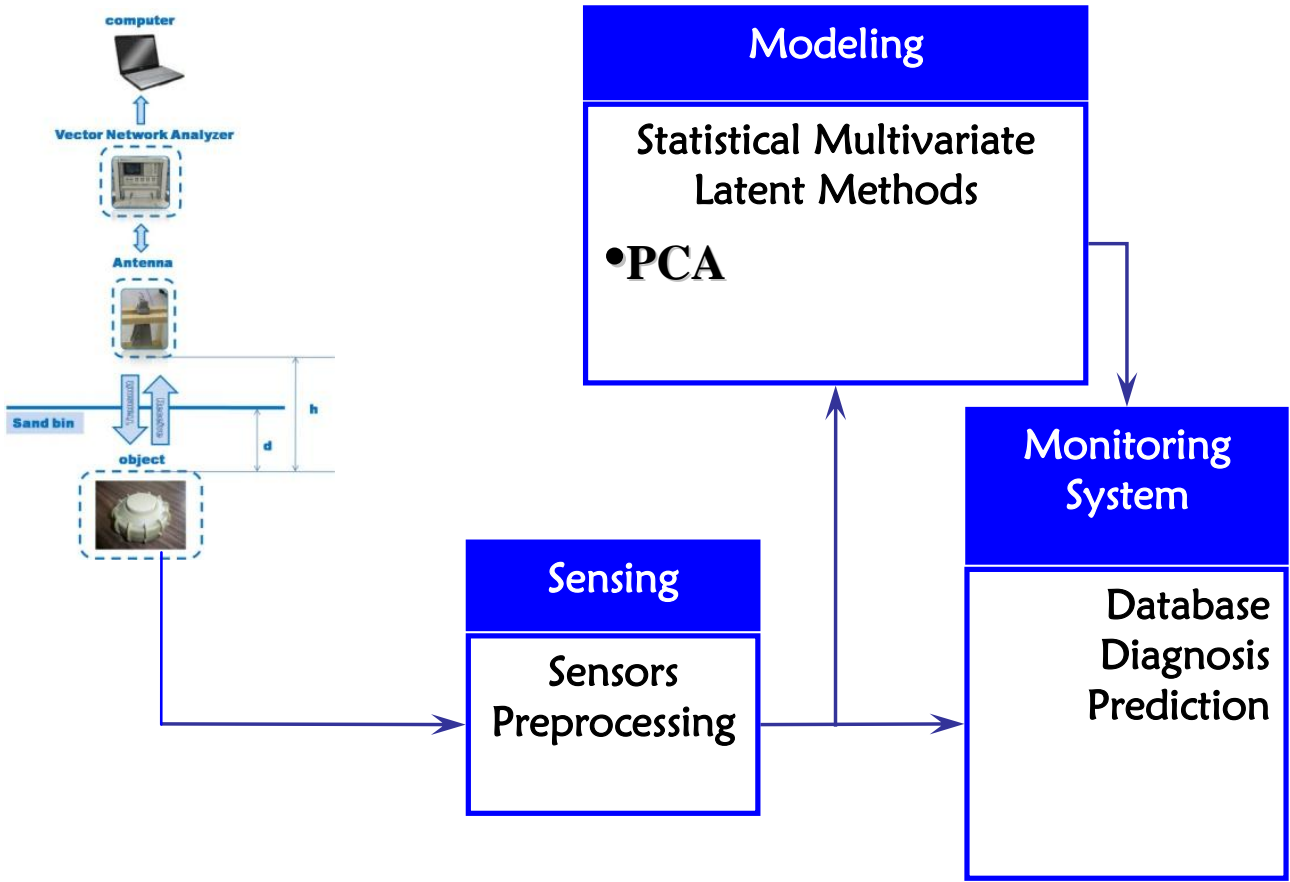

Fig. 5 Monitoring system of buried object location

The experimental work was conducted in two main folds. First, a total of 16 sensory positions (observations) were used to scan the sand box at different depths. Metal and plastic mines were buried at 3 different depths: 2, 5, and $10 \mathrm{~cm}$. Both the 3 depths (variables) and 16 observations construct the first "X" matrix. Second, more accurate measurements were made with a total of 208 positions (observations) with the same different depths (variables) for the metal and plastic mines generating the second " $\mathrm{X}$ " matrix. Model configuration for PCA is illustrated in the following sections. All the models were built using SIMCA-P code developed by Umetrics [11]. 
These variables represent the average of the reflected power-frequency domain records of the microwave antenna. Available groups of data are box with empty sand and box with buried metallic and plastic mine-like targets at different depths.

The PCA model is based on building classes or clusters using existing information inside the data (unsupervised learning). The model is established using a set of experimental runs called the training set that represents the sand box filled with sand. Finally, regression is made on the matrix $\mathrm{X}$ based on NIPALS algorithm.

To explore the collected data a first model using PCA was built using all the dataset by crossvalidation, now obtaining a two component model, with: R2X(cum): 0.92, Q2(cum) : 0.37.

Figure (6) illustrates a scatter plot of the two score vectors ( $\mathrm{t} 1$ and $\mathrm{t} 2$ ) of the PCA model using a set of 16 observations. It provides a clear vision of the dispersion of the data, with the sand data being discriminated against the buried metallic and plastic mine-like data along the first two components. This clustering is shown by manually highlighted with dotted circles in the t1 vs. t2 score plot.

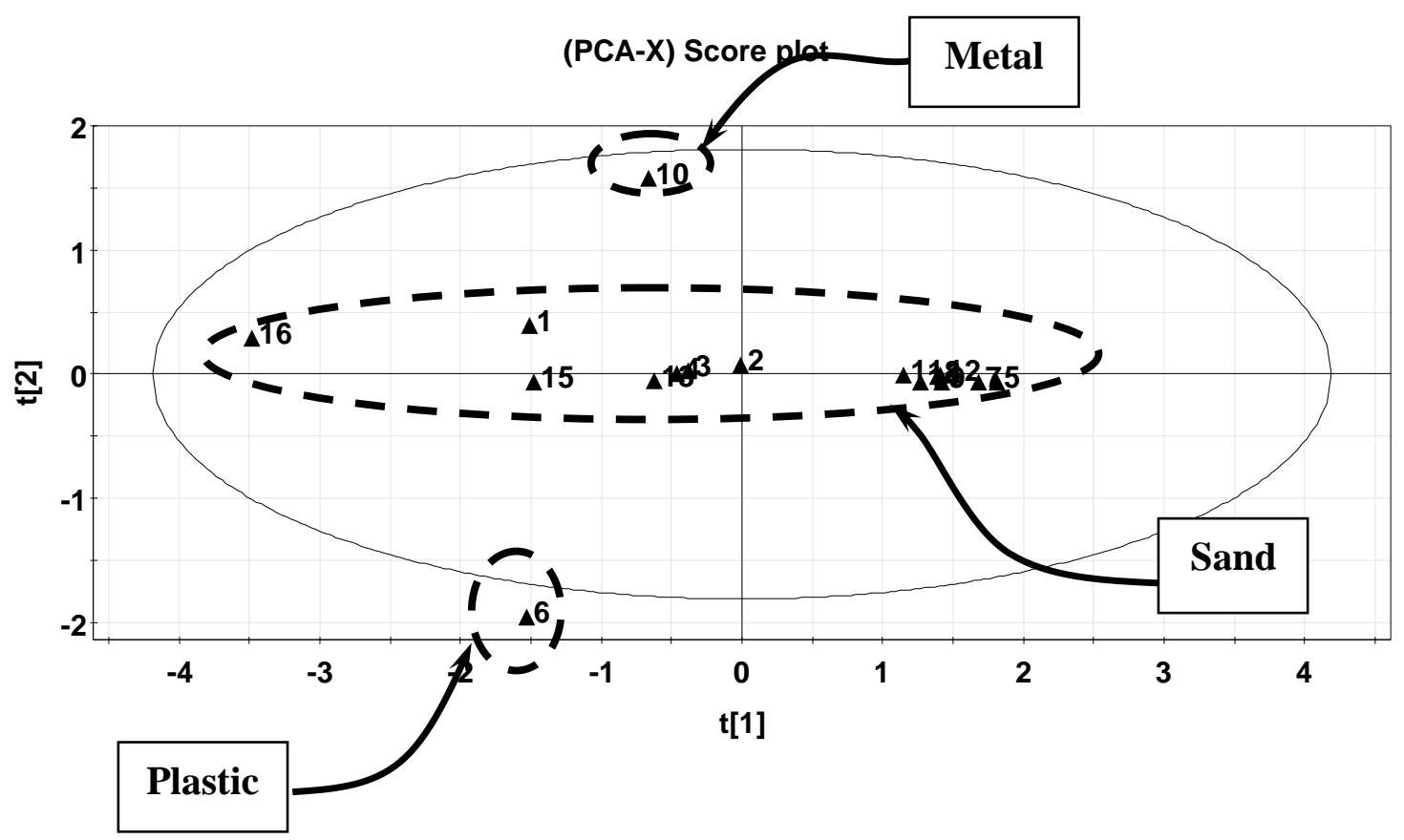

Fig. 6 Scatter plot of the two score vectors ( $t 1$ and $t 2)$ differentiate the data

By investigating the loading plot figure (7), one can detect that the metal object is dominating at the $2 \mathrm{~cm}$ depth (variable 1 in the direction of positive $\mathrm{t} 2$ ) whereas the plastic object has the same effect at the $5 \mathrm{~cm}$ depth (variable 2 in the direction of negative $\mathrm{t} 2$ ). In addition, deeper objects reflect less power resulting in less detectable objects, and this is clear by the third variable (near the $\mathrm{t} 1 \mathrm{axis}$ ) at $10 \mathrm{~cm}$ depth. 

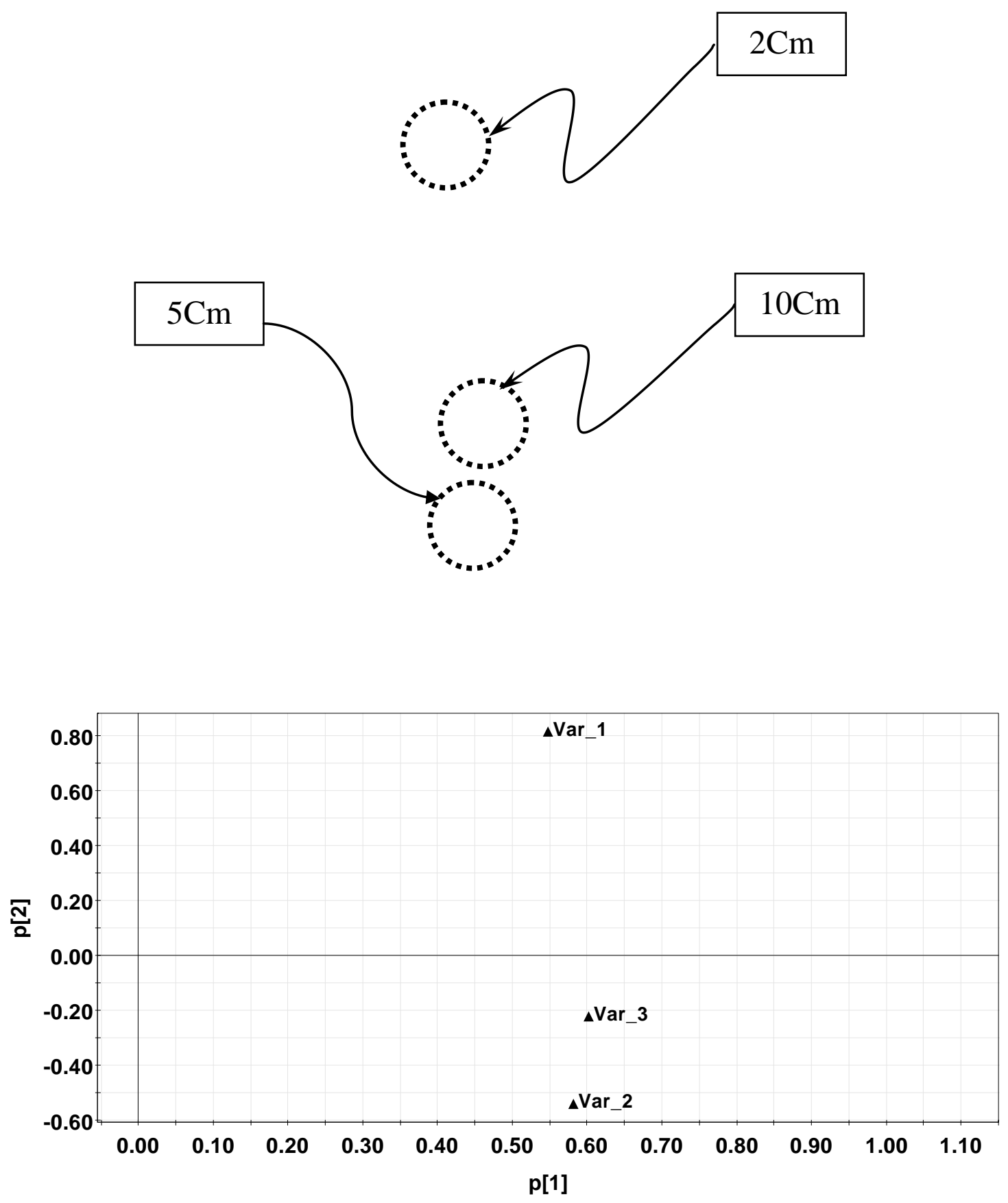

Fig. 7 Loading plot illustrate the dominating data

In this work, the main target is to build a monitoring system to detect and group different buried targets. Therefore, the same setting is repeated again with the use of data of the sand box as a training set. The model ends with 3 variables 14 observations and is fitted by crossvalidation, to get a two component model with: R2X(cum): 1, Q2(cum): 0.998. Figure (8) illustrates a scatter plot of the two score vectors ( $t 1$ and $\mathrm{t} 2$ ) of the PCA model. 
(PCA-X) Score plot

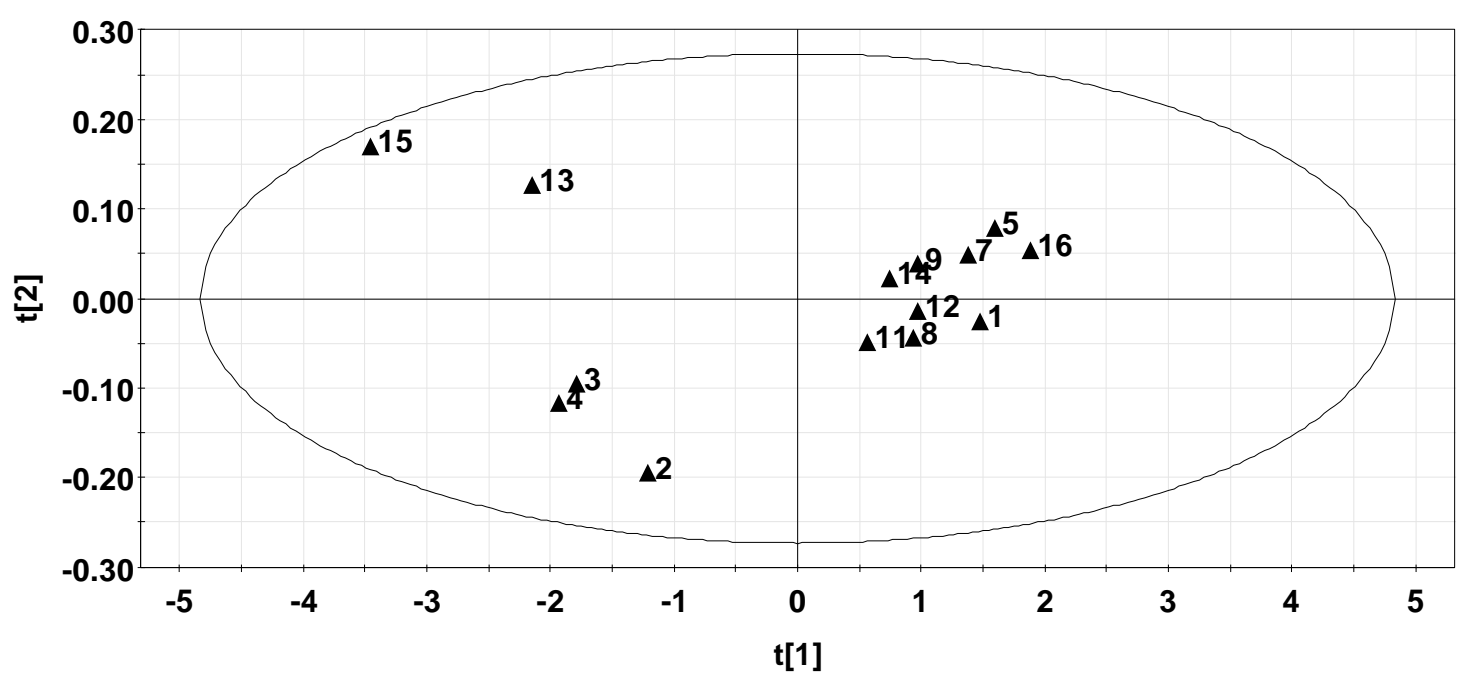

Fig. 8 Scatter plot of the two score vectors ( $t 1$ and $t 2)$ as a training set for monitoring

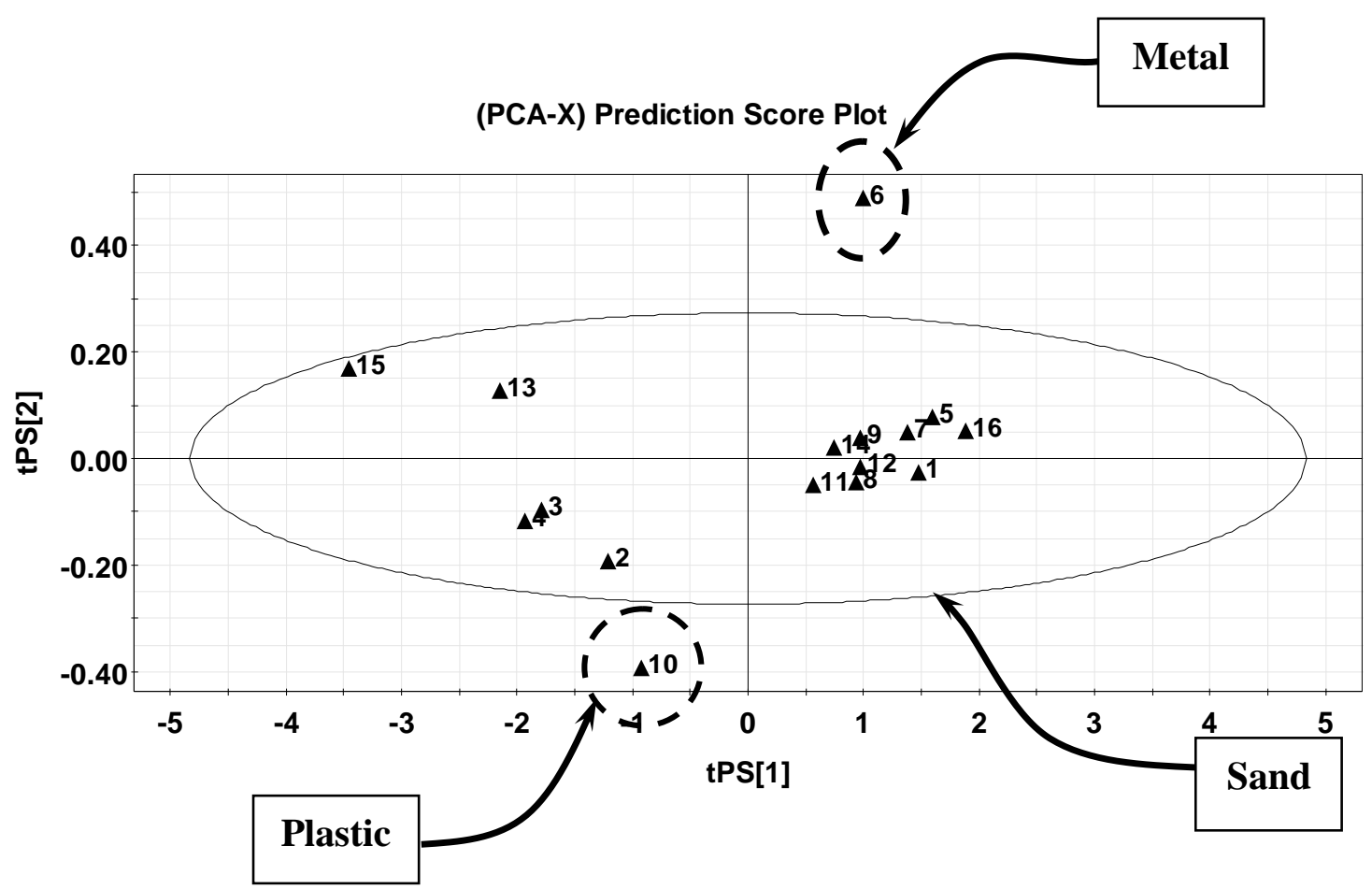

Fig. 9 PCA model used for validation

The developed PCA model was used on a validation set of the remaining 2 observations (1 plastic, 1 metal). The PCA scores values $(\mathrm{t} 1, \mathrm{t} 2)$ for these test observations are indicated by triangle points and manually highlighted with dotted circles in the $\mathrm{t} 1 \mathrm{vs}$. $\mathrm{t} 2$ score plot in figure (9). It can be seen from the figure that the PCA model succeeds in classifying the new observations sand, metal and plastic data. 
More investigation can be carried out using control charts. Figure (10) shows the Hotelling's $\mathrm{T}^{2}$ and DModX (model residual) plots with the test set. A clear advantage is realized from modeling the X-space over the present approaches. In case of the existence of buried metallic or plastic objects (not modeled) but similar to the same sand data, the Hotelling's $\mathrm{T}^{2}$ chart failed to detect this shift but is alarmed through the model residual DModX at points 6 (plastic) and 10 (metal).

16X3 finalplasMeta10cmonly.M2 (PCA-X), Untitled, PS-16X3 finalplasMeta10cmonly Shewhart (Subgroup 1): T2PS[Comp. 1]

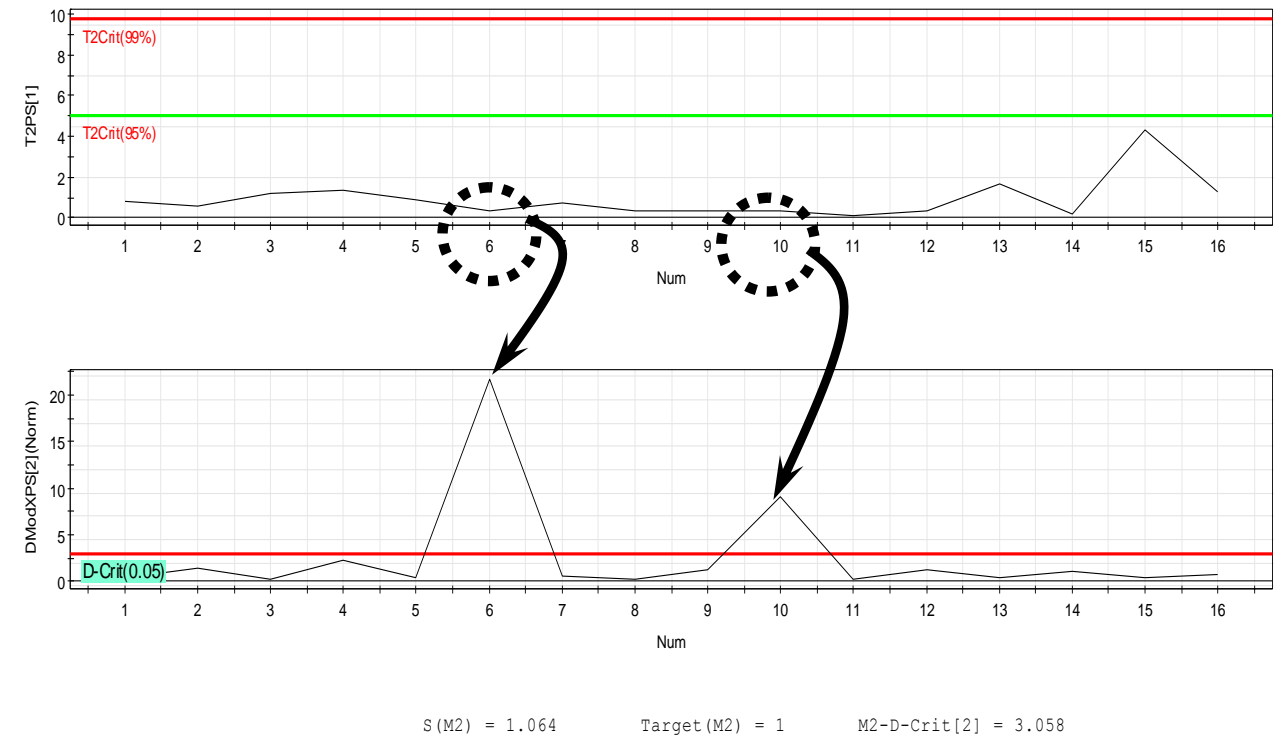

Fig. 10 Monitoring system built for alarm

The second model was based on 208 positions (observations) to scan the sand box at different depths. Metal and plastic mines were buried at 3 different depths: 2, 5, and $10 \mathrm{~cm}$. Both the 3 depths (variables) and 208 observations construct the second " $\mathrm{X}$ " matrix. To explore the collected data a first model using PCA was built using all the dataset by cross-validation, now obtaining a two component model, with: R2X(cum): 0.995, Q2(cum) : 0.629. Figure (11) illustrates a scatter plot of the two score vectors ( $t 1$ and $t 2$ ) of the PCA model using a set of 208 observations. It provides a clear vision of the dispersion of the data, with four main groups: sand, metallic object, plastic object and sand near the plastic box border. This clustering is shown by manually highlighted with dotted circles in the $\mathrm{t} 1 \mathrm{vs}$. $\mathrm{t} 2$ score plot. Aclear interference can be detected between two groups, the sand data near the box border (made from plastic) and the plastic buried mine-like target. To improve data grouping against buried object only, all data taken near the box border were removed. 


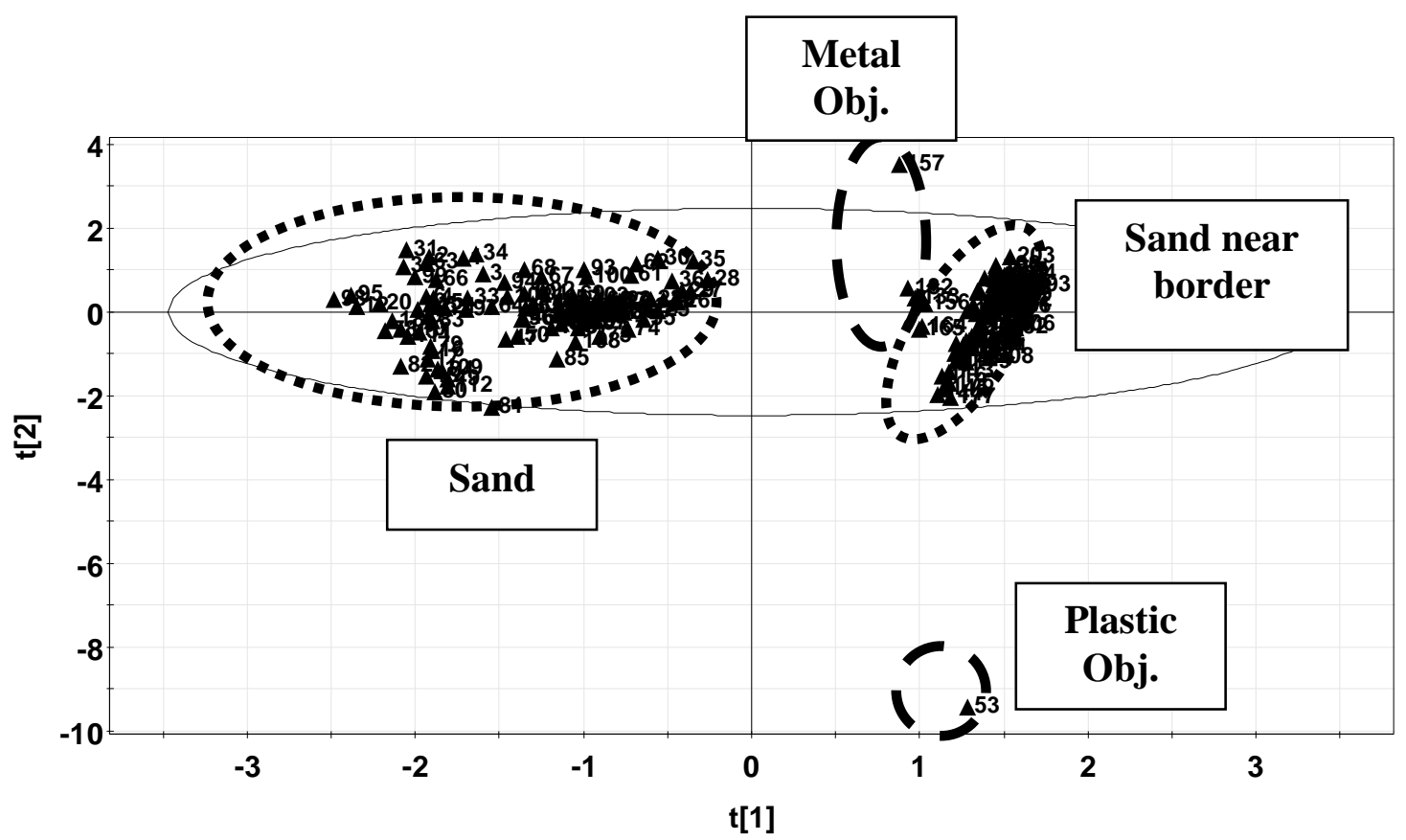

Fig. 11 Scatter plot of the two score vectors (t1 and t2) differentiate the data

A second model was built using the data of the sand as a training set. The model ends with 3 variables 106 observations and is fitted by cross-validation, to get a two component model with: R2X (cum): 1, Q2 (cum): 0.827. Figure (12) illustrates a scatter plot of the two score vectors ( $\mathrm{t} 1$ and $\mathrm{t} 2)$ of the PCA model.

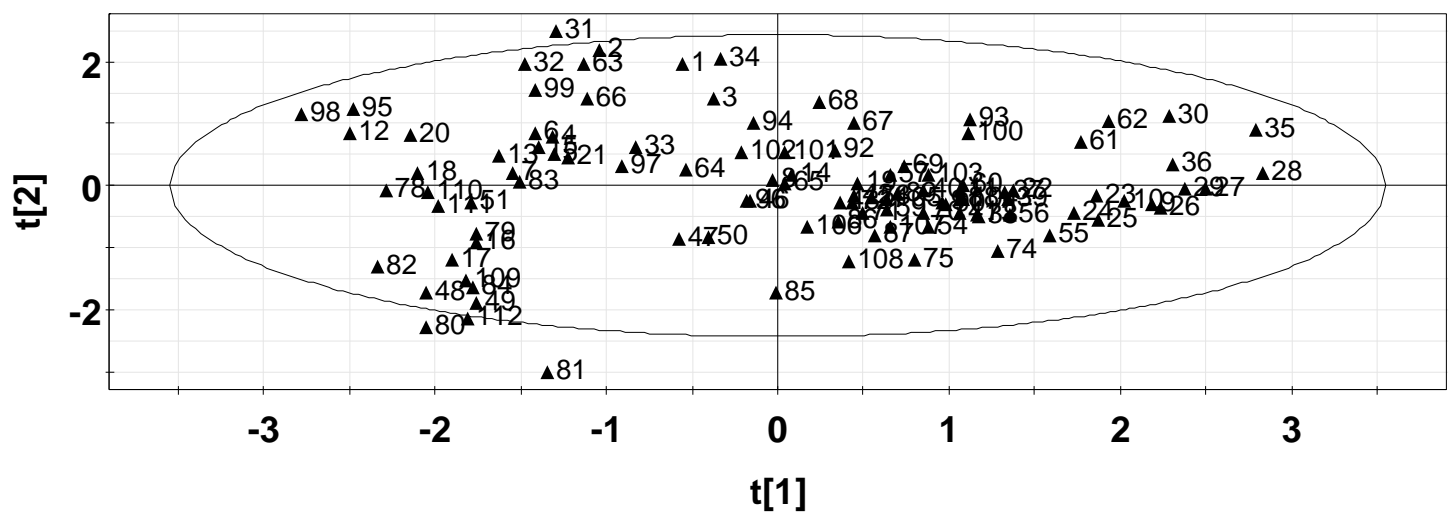

Fig. 12. Scatter plot of the two score vectors (t1 and t2) As a training set for monitoring

The developed PCA model was used on a validation set of the remaining 12 observations (6 plastic, 6 metal). It can be seen from the figure that the PCA model succeeds in grouping the new observations outside the confidence interval. Also, metal and plastic mine-like target data can be clustered by the manually highlighted dashed line as shown in figure (13). 


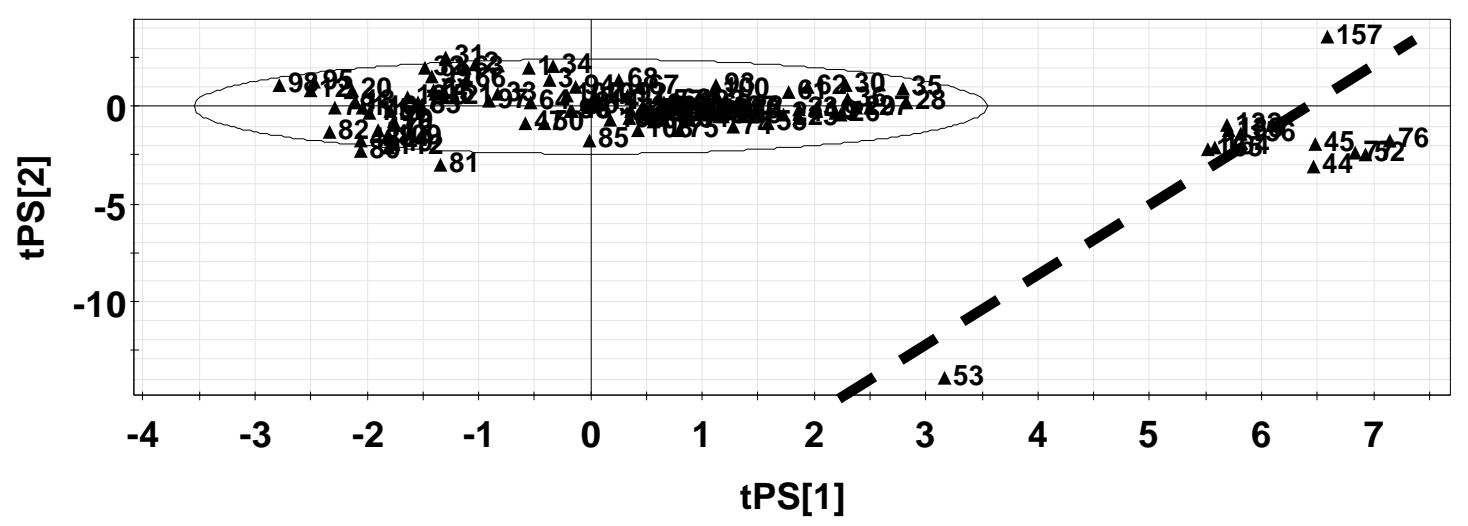

Fig. 13 PCA model used for validation

\section{Conclusions \& Future Work}

Multivariate projection techniques are statistical methods, which can be used to describe, analyze and model multivariate data, while simultaneously measuring several predictors and response variables. The application of multivariate techniques to detect mine-like target and to discriminate between plastic and metal objects are the main contributions of this research. The approach uses experimental data for model building and testing. Principal Component Analysis (PCA) has been used for processing the data. This technique is effective for the clustering of different groups. Results show that the proposed technique can successfully differentiate between metallic and plastic mines-like targets buried in dry soil.

The current work will be extended to include variable soil types and the decision making algorithm would be modified to be based on implication in place of association. That will need automatic detection algorithms for GPR.

\section{References}

[1] The United Nations Mine Action Services, http:_www.un.org_Depts_dpko_mine

[2] Sieber, A., 1995, Localization and identification of anti-personnel mines, European Commission Joint Research Center International Workshop.

[3] T.R. Witten, Present state of the art in ground-penetrating radars for mine detection, in: Proceedings of the SPIE Conference, vol. 3392, Orlando, FL, 1998, pp. 576-586.

[4] P.D. Gader, M. Mystkowski, Y. Zhao, Landmine detection with ground penetrating radar using hidden Markov models, IEEE Trans. Geosci. Remote Sensing 39 (6) (June 2001) 1231-1244.

[5] P. Torrione, L. Collins, F. Clodfelter, S. Frasier, I. Starnes, Application of the LMS algorithm to anomaly detection using the Wichmann/Niitek ground penetrating radar, in: Proceedings of the SPIE Detection and Remediation Technologies for Mines and Minelike Targets, vol. VIII, Orlando, April 2003, pp. 1127-1136.

[6] S. Yu, R.K. Mehra, T.R. Witten, Automatic mine detection based on ground penetrating radar, in: Proceedings of SPIE, Detection and Remediation Technologies for Mines and Minelike Targets, vol. IV, Orlando, FL, April 1999, pp. 961-972.

[7] J. J. Ding, "A Linear Regression Model for the Identification of Unbalance Changes in Rotating Machines", Journal of Sound and vibration, 231(1), pp.125-144, 2000. 
[8] Zeki Kiral and Hira Karagulle, "Vibration analysis of rolling element bearings with various defects under the action of an unbalanced force", J. Mechanical Systems and Signal Processing 20, pp. 1967-1991, 2006.

[9] Wilson q. Wang, Fathy Ismail and M. Farid Golnaraghi, "Assessment of Gear Damage Monitoring Techniques Using Vibration Measurements”, J. Mechanical Systems and Signal Processing 15(5), pp. 905-922, 2001.

[10] Yimin Zhan and Viliam Makis, "A robust diagnostic model for gearboxes subject to vibration monitoring", Journal of Sound and Vibration 290, pp. 928-955, 2006.

[11] MacGregor J.F., Yu H., Munoz S. and Cerrillo J., "Data-based latent variable methods for process analysis, monitoring and control", Journal of Computers and Chemical Engineering 29, 1217-1223, 2005.

[12] P. Nomikos and J. MacGregor, "Monitoring batch processes using multiway principal component analysis", AIChE J., Vol.40, 1361, 1994.

[13] Eriksson L., Johansson E., Wold N.K. and Wold S., "Multi- and Megavariate data analysis, principles and applications"; Umetrics AB, Sweden, 2006.

[14] Kourti T. and MacGregor J. F., "Multivariate SPC methods for process and product monitoring", Journal of quality technology, Vol.28, 4, October, 1996.

[15] Wold S., Esbensen K. and Geladi P., "Principal component analysis", Chemometrics and intelligent laboratory systems, 2: 37-52, 1987.

[16] Jackson J.E., “A User's Guide to Principal Components”, Wiley, New York, 1991.

[17] Wold S., Sjostrom M. and Eriksson L., "PLS-regression: a basic tool of chemometrics", Chemometric and intelligent laboratory systems, 58:109-130, 2001.

[18] Lennox B., Hiden H.G., Montague G.A., Kornfeld G. and Goulding P.R., "Application of multivariate statistical process control to batch operation", J. Computers and chemical engineering, V24, n2, July, PP. 291-296, 2000.

[19] Goulding P.R., Lennox B., Sandoz D.J., Smith K.J. and Marjanovic, "Fault detection in continuous processes using multivariate statistical methods", International journal of systems science, 31(11), pp. 1459-1471, 2000.

[20] Yon S., Landry J., Wold N.K., Pepe and Wold S., "Multivariate process monitoring and early fault detection (MSPC) using PCA and PLS", Plant automation and decision support conference, September 21-24, San Antonio, Texas, 2003.

[21] Chen J. and Liu K.C., "On-line batch process monitoring using dynamic PCA and dynamic PLS models", J. Chemical engineering science 57:63-75, 2002.

[22] Kourti T., "Abnormal situation detection, three-way data and projection methods robust data archiving and modeling for industrial applications", Annual reviews in control 27: 131-139, 2003.

[23] P. Nomikos and J. MacGregor, "Monitoring batch processes using multiway principal component analysis", AIChE J., Vol.40, 1361, 1994.

[24] Jolliffe, I.T., "Principle component analysis", springer virlag, New York, 1986. 